\section{$\gamma_{8}$}

${ }^{1}$ Department of Ear, Nose \&
Throat (ENT)-Head \& Neck Surgery, Ng Teng Fong General Hospital, Singapore, Singapore ${ }^{2}$ Department of Pathology, National University Hospital, Singapore, Singapore ${ }^{3}$ Division of Respiratory and Critical Care Medicine, University Medicine Cluster, National University Hospital, Singapore, Singapore

\section{Correspondence to}

Dr Chew Lip Ng, Department of Ear, Nose \& Throat (ENT)-Head \& Neck Surgery, Ng Teng Fong General Hospital, Singapore S609606, Singapore: chew_lip_ng@nuhs.edu.sg

Received 18 September 2017 Revised 29 December 2017 Accepted 5 January 2018 Published Online First 26 January 2018

\section{Check for updates}

To cite: $\mathrm{Ng} \mathrm{CL}$, Liu $\mathrm{X}$, Tan NJH, et al. Thorax 2018:73:693-694.

\title{
Pulmonary melioidosis with laryngeal involvement: an unusual site of infection
}

\author{
Chew Lip Ng, ${ }^{1}$ Xuandao Liu, ${ }^{1}$ Nicholas Jin Hong Tan, ${ }^{2}$ Vyas Malur Narayan Prasad, \\ Pyng Lee ${ }^{3}$
}

A 42-year-old Malay man presented with cough productive of yellowish sputum, hoarseness and intermittent daily fever associated with chills and rigours for 3 weeks. Apart from previous pulmonary TB in 1994 for which treatment was complete, there were no other comorbidities. The patient was an active cigarette smoker of 20 pack-years, and worked as an outdoor project coordinator frequently in contact with soil during field surveys in gardens. Chest radiograph showed right perihilar consolidation (figure 1A). CT thorax showed mass-like consolidation of the right upper lobe with extensive mediastinal lymphadenopathy (figure 1B). White cell count was 8.8 (range $3.4-11.0 \times 10^{9} / \mathrm{L}$ ) with neutrophilia $72.2 \%$; blood, sputum and urine for bacterial cultures, as well as sputum for acid fast bacilli smears and TB PCR assay, were negative. Random blood sugar level was $23.0 \mathrm{mmol} / \mathrm{L}$ (range

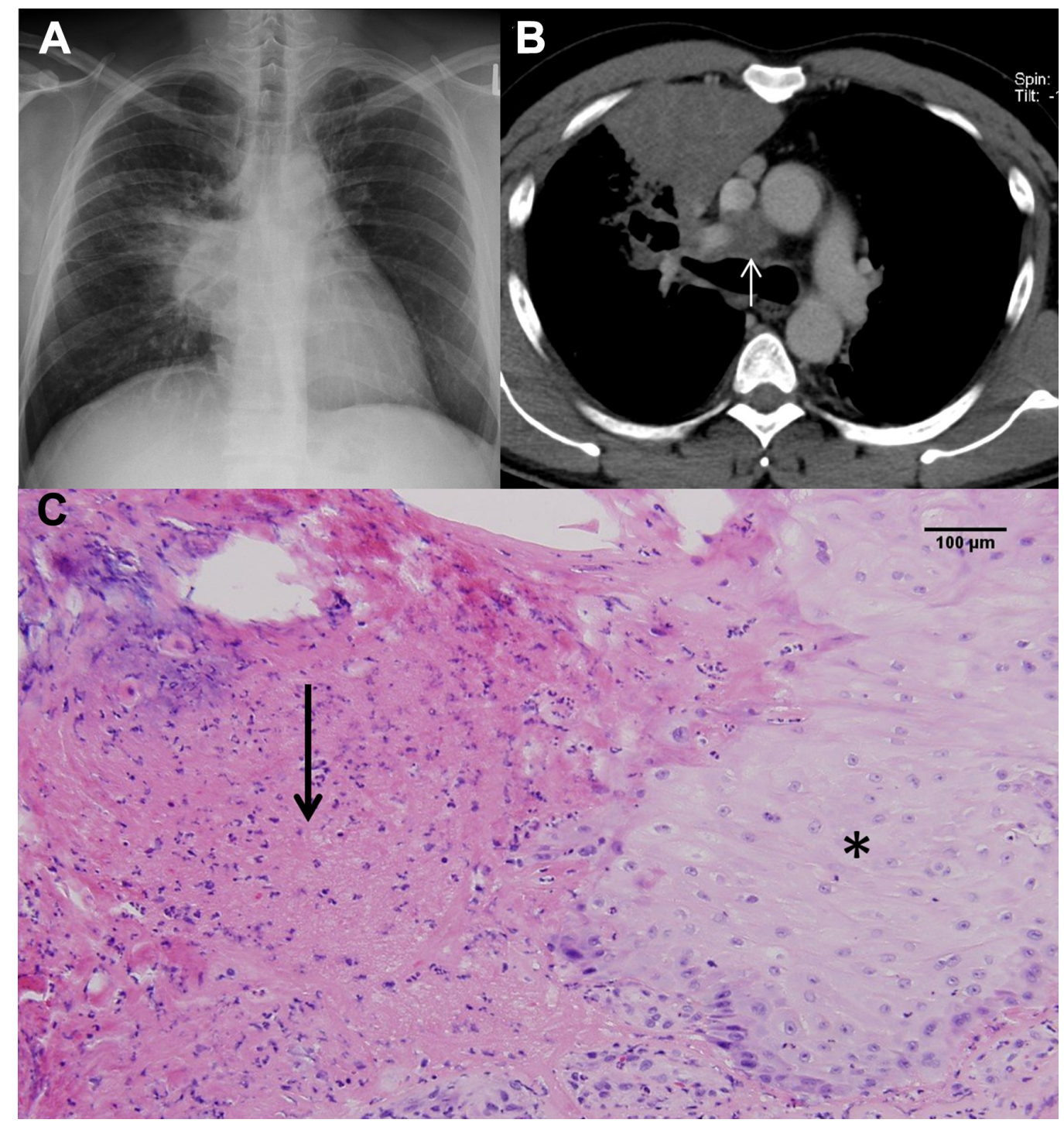

Figure 1 (A) Chest radiograph showing right perihilar consolidation. (B) CT thorax, axial view, showing consolidation of the right upper lobe and mediastinal lymphadenopathy (white arrow). (C) Histology of vocal fold biopsy showing superficial squamous mucosa (asterisk) demonstrating reactive changes with ulceration and fibrinous exudate (black arrow) without granulomatous lesions (H\&E, 200x). 


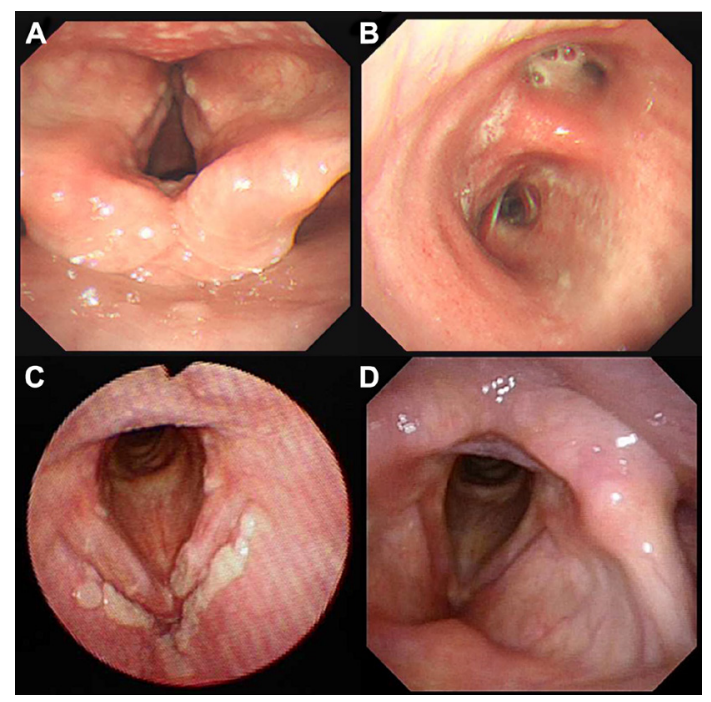

Figure 2 Progression of laryngeal infective ulcers. (A, B) Day 2 of admission. Bronchoscopic image of larynx showing nodules over both false folds; purulent secretions from right upper lobe on bronchoscopy. (C) Day 5 of admission. Prominent ulcers over both false vocal folds and anterior third of both true vocal folds. (D) Five months since date of admission, having completed 6 weeks of intravenous ceftazidime and 12 weeks of oral co-trimoxazole. Ulcers completely resolved with no residual scarring.

4.0-7.8 mmol/L) and glycated haemoglobin level was $12.1 \%$. Bronchoscopy showed mobile vocal folds, ulcers affecting the false and anterior third of true vocal folds with whitish nodules that extended to the subglottis and upper trachea (figure 2A-C). From bronchoalveolar lavage specimen, Burkholderia pseudomallei, sensitive to ceftazidime, co-trimoxazole, doxycycline and imipenem, was isolated. Laryngoscopy and biopsy of left vocal fold lesions revealed superficial squamous mucosal ulceration with reactive epithelial changes without dysplasia, malignancy or granulomatous inflammation (figure $1 \mathrm{C}$ ). The patient received intravenous ceftazidime for 6 weeks, followed by oral co-trimoxazole for 6 months. Good diabetic control was achieved with dietary adjustment and oral metformin. The patient experienced radiological resolution as well as voice recovery after 3 months of therapy. Repeat endoscopy at the end of treatment showed normal larynx (figure 2D). There was no relapse of symptoms at 1 year of follow-up.
Melioidosis is an infectious disease caused by $B$. pseudomallei. Pneumonia, genitourinary infections and skin and soft-tissue abscesses are common manifestations in adults. ${ }^{1}$ Infection arises from direct inoculation from skin contact with soil followed by haematogenous spread or inhalation. In our patient, laryngeal involvement from contiguous spread was the most likely mechanism as there were superficial ulcerations over the anterior third of the true vocal fold and medial edges of the false vocal folds, which represented sites of epithelial trauma during vocal fold adduction. This process may be akin to laryngeal TB where expectoration of bacilli-laden endobronchial secretions leads to direct invasion of chronically irritated laryngeal mucosa. ${ }^{2}$ Thus, epithelial trauma could explain for the ulcers observed over the vocal folds but not of the upper trachea. Histology of melioidosis is non-specific ${ }^{3}$ and biopsy of vocal cord lesions showed ulceration of the squamous mucosa with fibrinopurulent exudates. We describe the first reported case of laryngeal melioidosis in a patient with pulmonary infection and type 2 diabetes mellitus. Early recognition and appropriate antibiotic therapy against $B$. pseudomallei leads to complete resolution of laryngeal lesions and good recovery of function.

Contributors CLN and XL: conception of the article, literature search, analysis and interpretation of data, drafting the article, revising it critically for important intellectual content, and final approval of the version to be published. NJHT: literature search, analysis and interpretation of data, drafting the article, revising it critically for important intellectual content, and final approval of the version to be published. VMNP and PL: conception of the article, literature search, analysis and interpretation of data, revising it critically for important intellectual content, and final approval of the version to be published.

Funding This research received no specific grant from any funding agency in the public, commercial or not-for-profit sectors.

Competing interests None declared.

Patient consent Obtained.

Provenance and peer review Not commissioned; externally peer reviewed.

(c) Article author(s) (or their employer(s) unless otherwise stated in the text of the article) 2018. All rights reserved. No commercial use is permitted unless otherwise expressly granted.

\section{REFERENCES}

1 Cheng AC, Currie BJ. Melioidosis: epidemiology, pathophysiology, and management. Clin Microbiol Rev 2005;18:383-416.

2 Levenson MJ, Ingerman M, Grimes C, et al. Laryngeal tuberculosis: review of twenty cases. Laryngoscope 1984;94:1094-7.

3 Wong KT, Puthucheary SD, Vadivelu J. The histopathology of human melioidosis. Histopathology 1995:26:51-5. 